\title{
METODOLOGIA PARA ANÁLISE DA DEPENDÊNCIA TÉRMICA DA GERMINAÇÃO PELO MODELO DE GRAUS-DIA
}

\section{Victor José Mendes Cardoso}

Unesp, Instituto de Biociências, Departamento de Botânica, Av. 24-A, 1515, Caixa Postal 199, Rio Claro, SP, Brasil, CEP: 13506-900.

E-mail: victorjc@rc.unesp.br

\section{RESUMO}

Inúmeros trabalhos científicos tratam dos efeitos da temperatura sobre a velocidade de processos biológicos, entre eles a germinação. A análise do efeito térmico pode ser feita a partir de índices numéricos ou pela análise das curvas de germinação, comumente apresentadas como as porcentagens acumuladas de germinação contra os respectivos tempos. Tais curvas, passíveis de descrição por modelos empíricos, em geral representam melhor o comportamento das sementes ao longo do tempo do que o fazem índices isolados. Por outro lado, na análise das curvas de germinação, uma questão que se apresenta é a atribuição de significados biológicos aos parâmetros das equações derivadas desses modelos. Assim, é desejável que, além de descrever da maneira mais adequada possível uma curva, os parâmetros do modelo utilizado tenham algum significado biológico, o que muitas vezes não ocorre. Considerando-se o envolvimento de populações e porcentagens em experimentos de germinação, um modelo de análise que vem sendo bastante utilizado nas duas últimas décadas é aquele baseado no conceito de graus-dia. Este artigo trata da base conceitual e apresenta os procedimentos para aplicação do modelo de graus-dia em estudos de germinação, usando-se o método da regressão probítica, tanto em temperaturas infra-ótimas como supra-ótimas.

Palavras-chave: Tempo térmico; temperatura; sementes; modelagem; revisão.

\begin{abstract}
METHODOLOGY FOR ANALYSIS OF THE THERMAL DEPENDENCE OF GERMINATION BY THERMAL-TIME MODEL. The effects of temperature on the rate of biological processes such as the germination are reported in several papers. The analysis of the thermal effect can be performed either from numerical indices or germination curves, which is usually presented as the accumulated percentage of germination versus time. Such curves can be fitted by empirical models and generally they offer a better description of the time-course of germination than single-value indices. On the other hand, in analyzing the germination curves, a question arises whether the model parameters have biological meaning. Thus, it is desirable that the model parameters both describe properly the germination curve and have biological meaning, which often does not occur. Considering the involvement of populations and percentages in germination assays, an analytical model has been widely used in the last two decades which is based on the concept of thermaltime. This article discusses the conceptual basis and provides procedures for applying the thermal-time model in germination studies by using the probit regression method, both at infra and supra-optimal temperatures.
\end{abstract}

Keywords: Thermal time; temperature; seeds; modeling; review.

\section{RESUMEN}

METODOLOGIA PARA EL ANÁLISIS DE LA DEPENDENCIA TÉRMICA DE LA GERMINACIÓN POR MEDIO DE UN MODELO GRADOS DIA. Numerosos trabajos científicos reportan los efectos de la temperatura sobre la velocidad de los procesos biológicos, entre ellos la germinación. El análisis del efecto térmico puede ser realizado a partir de índices numéricos o mediante el análisis de curvas de germinación, comúnmente presentadas como los porcentajes acumulados de la germinación contra los 
respectivos tiempos. Tales curvas, que pueden ser descriptas por modelos empíricos, generalmente representan mejor el comportamiento de las semillas en el transcurso del tiempo de lo que lo hacen los valores individuales de los índices. Por otro lado, en el análisis de las curvas de germinación, surge una pregunta acerca de la atribución de significados biológicos a los parámetros de las ecuaciones derivadas de esos modelos. De este modo, sería apropiado que los parámetros del modelo describan adecuadamente la curva de germinación y que tengan un significado biológico, algo que a menudo no ocurre. Teniendo en cuenta la participación de las poblaciones y porcentajes en los ensayos de germinación, un modelo analítico ha sido ampliamente utilizado en las últimas dos décadas basados en el concepto térmico-tiempo (grados día). Este artículo discute la base conceptual y presenta procedimientos de aplicación del modelo grados día en estudios de germinación, utilizando el método de regresión probit, tanto en condiciones de temperaturas infra-óptimas como supraóptimas.

Palabras clave: Tiempo térmico; temperatura; semillas; modelado; revisión.

\section{INTRODUÇÃO}

A germinação, ou seja, a retomada das atividades metabólicas e do crescimento do embrião em sementes maduras de espermatófitas separadas da planta mãe, depende de um conjunto de condições ambientais, entre elas a temperatura (Labouriau 1983, Cardoso 2008). A temperatura é um fator ao qual uma semente - como qualquer sistema vivo - está permanentemente exposta, constituindo-se assim num forte indicador do ambiente operacional (fatores ambientais que afetam diretamente o sistema) e de sua variação a longo e curto prazo. A resposta à temperatura tem sido caracterizada pelas chamadas 'temperaturas cardeais', que são as temperaturas mínima $\left(\mathrm{T}_{\mathrm{b}}\right)$, máxima $\left(\mathrm{T}_{\mathrm{c}}\right)$ e ótima $\left(\mathrm{T}_{\mathrm{o}}\right)$ para ocorrência do processo. $\mathrm{T}_{\mathrm{o}}$ representa a temperatura ou intervalo térmico onde a germinação é máxima e uniforme, enquanto que $T_{b}$ e $T_{c}$ correspondem aos limites térmicos extremos a partir dos quais o processo deixa de ocorrer (Labouriau 1983). No intervalo delimitado por $T_{b} e$ $\mathrm{T}_{\mathrm{c}}$ a temperatura afeta não apenas a germinabilidade (capacidade de germinação averiguada pela porcentagem final de sementes germinadas) como também - e principalmente - a velocidade (recíproca dos tempos de germinação, 1/t) (Labouriau 1972). $\mathrm{Na}$ maioria dos casos, a velocidade de germinação aumenta com a temperatura no intervalo entre $T_{b} e$ $\mathrm{T}_{\mathrm{o}}$ (faixa infra-ótima) e diminui entre $\mathrm{T}_{\mathrm{o}}$ e $\mathrm{T}_{\mathrm{c}}$ (faixa supra-ótima). Questões como: o que determina as temperaturas cardeais; ou, por que a distribuição dos tempos de germinação de sementes individuais varia em função da temperatura, tem importância não só do ponto de vista do conhecimento da ecofisiologia de uma espécie, como também do conhecimento da fisiologia do processo de germinação e de seus fatores limitantes (Labouriau 1970, 1983).

Considerando-se que a germinação representa uma etapa fundamental e crítica para a sobrevivência da maioria das fanerógamas, o homem busca entender esse processo para melhor controlá-lo, seja interferindo diretamente na sua ocorrência, seja prevendo o comportamento germinativo de populações. Essa capacidade de previsão de um determinado fenômeno biológico passa pela elaboração de modelos que possibilitem uma quantificação da resposta do sistema vivo a flutuações do meio. O principal problema nesse tipo de estudo decorre exatamente da contínua flutuação de fatores como a temperatura e água no ambiente natural, expondo assim a semente a um amplo espectro de condições do micro-ambiente. Na busca de modelos de caráter mais ou menos geral que tentem explicar a resposta do sistema a tais flutuações, pesquisadores recorrem a ensaios em condições controladas, mais facilmente interpretáveis (e tratáveis) do ponto de vista matemático. Modelos baseados em parâmetros populacionais, como graus-dia (thermal time), são ferramentas que permitem estimar, a partir de experimentos relativamente simples em condições controladas, o tempo necessário para a germinação de uma semente em um dado regime térmico com base em alguns poucos parâmetros retirados de tais ensaios (Meyer et al. 2000, Bradford 2002). Este artigo trata da fundamentação teórica do modelo de graus-dia, bem como dos procedimentos para sua aplicação em estudos de germinação e crescimento inicial de plantas. 


\section{O CONCEITO DE GRAUS-DIA}

Considerando-se que a temperatura afeta a velocidade de crescimento do organismo, e considerando-se que existem limites térmicos a partir dos quais não há crescimento, ou este é bastante reduzido (Figura 1), um ser vivo deve requerer uma determinada quantidade de calor (energia) para passar de um determinado estádio de desenvolvimento a outro. Essa quantidade de calor obedece a um princípio de reciprocidade, ou seja, ela representa o produto da temperatura $(\mathrm{T})$ pelo tempo de exposição (t) e seria constante para cada organismo. Em outras palavras, a quantidade de calor para um organismo alcançar certo estádio de desenvolvimento não varia, de modo que o produto da temperatura (dentro dos limites representados pelas temperaturas cardeais extremas) pelo tempo deverá representar um 'tempo corrigido' - também conhecido como 'tempo fisiológico' ou 'tempo térmico' (thermal time) - para esse organismo chegar a certo tamanho. O 'tempo térmico' é frequentemente expresso em unidades chamadas graus-dia $\left({ }^{\circ} \mathrm{D}\right)$, constituindo-se num referencial para se comparar a resposta de diferentes organismos à temperatura.
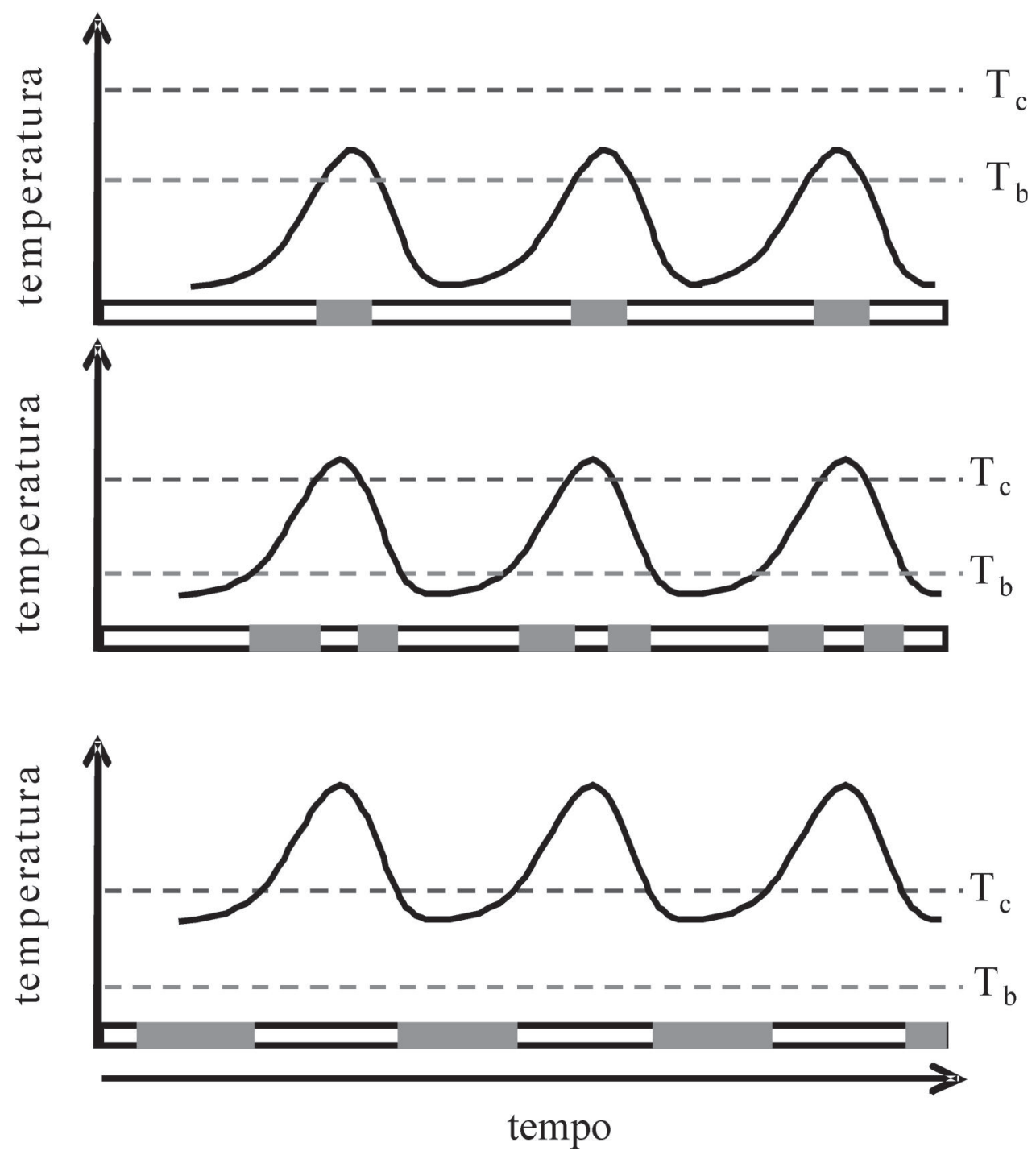

Figura 1. Três possíveis relações entre o regime de flutuação térmica diária (linha contínua) e os limites térmicos superiores $\left(\mathrm{T}_{c}\right)$ e inferiores $\left(\mathrm{T}_{\mathrm{b}}\right)$, acima e abaixo dos quais não há crescimento. Os segmentos cinza no eixo x indicam as condições térmicas em que teoricamente há crescimento. Figura adaptada de UC IPM (2003).

Figure 1. Three relationships possibilities amongst daily thermal fluctuation (continuous line) and both the upper $\left(T_{d}\right)$ and lower $\left(T_{b}\right)$ thermal thresholds, above and below which the growth stops. The grey areas on X-axis show the periods in which there is growth. Figure adapted from UC IPM (2003). 
Determinando-se (a partir de ensaios preliminares) a temperatura base $\left(\mathrm{T}_{\mathrm{b}}\right)$ como sendo, digamos, $5^{\circ} \mathrm{C}$ para uma determinada resposta biológica como, por exemplo, o crescimento da parte aérea de plântulas de uma determinada espécie, pode-se estimar a quantidade de 'tempo térmico' necessário para que um dado estádio de desenvolvimento seja alçando, tomando-se como referência $T_{b}$ e as temperaturas médias diárias previstas e/ou medidas (T). Por exemplo, assumindo-se que durante um período de oito dias foram registradas temperaturas médias diárias como aquelas apresentadas na Tabela 1 , ao final desses oito dias teremos uma quantidade acumulada de 33 graus-dia que representam o tempo ou soma térmica, lembrando sempre que isso é em relação à $\mathrm{T}_{\mathrm{b}}$ para a variável escolhida (no caso, o crescimento da parte aérea). Supondo-se que, com base em experimentos anteriores em condições controladas, essa quantidade de graus-dia seja suficiente para que $50 \%$ das plântulas atinjam, por exemplo, $2 \mathrm{~cm}$ de altura, nessas condições o agricultor deverá esperar seis dias para que metade de sua cultura tenha esse tamanho. Numa outra condição, se as temperaturas do ambiente forem mais elevadas, essa quantidade de 'tempo térmico' $\left(33^{\circ} \mathrm{D}\right)$ deverá ser alcançada num intervalo de tempo menor, digamos, três dias, evidentemente desde que os níveis de água no solo sejam semelhantes. Desse modo, partindo-se da hipótese de que cada estádio de desenvolvimento de um organismo requer uma quantidade determinada de graus-dia, a utilização desse 'tempo corrigido' deve permitir a previsão de quando um estádio prédeterminado de desenvolvimento será alcançado. Por outro lado, esse modelo não deve funcionar igualmente para todo o intervalo térmico situado entre as temperaturas cardeais extremas do referido estádio, devendo-se levar em conta se o regime térmico está circunscrito à faixa térmica infra-ótima ou se engloba também a faixa supra-ótima. No primeiro caso, em que a velocidade do processo (V) aumenta linearmente com a temperatura, o acúmulo de 'tempo térmico' $(\theta)$ é mais observável, ao passo que na região supra-ótima - onde $\mathrm{V}$ tende a decair com o aumento da temperatura, a aplicação do modelo se torna mais difícil. De fato, os métodos usualmente utilizados para se estimar o acúmulo de graus-dia incluem apenas a temperatura base entre os parâmetros (Maniero 1980), ignorando $T_{c}$ ou os efeitos causados por temperaturas supra-ótimas. No caso do processo de germinação (protrusão radicular, segundo o critério biológico), alguns modelos partem do pressuposto que a quantidade de $\theta$ aumenta apenas na faixa infraótima, atingindo um máximo em $\mathrm{T}_{\mathrm{o}}$ e permanecendo constante a partir daí (Alvarado \& Bradford 2002). Possíveis fatores limitantes da germinação na faixa supra-ótima serão discutidos adiante.

Tabela 1. Acúmulo de 'tempo térmico' ao longo de um período de oito dias numa condição hipotética em que a temperatura mínima para ocorrência do processo $\left(\mathrm{T}_{\mathrm{b}}\right)$ equivale a $5^{\circ} \mathrm{C}$, onde $\mathrm{T}$ indica a temperatura do ambiente. Note que para os casos em que $\mathrm{T}<\mathrm{T}_{\mathrm{b}}$ não se computa acúmulo de 'tempo térmico'.

Table 1. Accumulated 'thermal time' over eight days with the minimum temperature for the process occurrence $\left(T_{b}\right)$ set at $5^{\circ} \mathrm{C}$, where $T$ indicates the temperature. Note that for $T<T_{b}$ 'thermal time' do not accumulate.

\begin{tabular}{|c|c|c|c|c|c|}
\hline $\begin{array}{l}\text { tempo }(\mathrm{t}) \\
\quad(\text { dias })\end{array}$ & $\begin{array}{l}\text { intervalo de } \\
\text { tempo }(\Delta \mathrm{t})\end{array}$ & $\mathrm{T}\left({ }^{\circ} \mathrm{C}\right)$ & $\mathrm{T}_{\mathrm{b}}$ & $\begin{array}{c}\text { soma ou 'tempo térmico' ( } \mathrm{T}-\mathrm{T} \\
\left.{ }_{\mathrm{b}}\right) \cdot \Delta \mathrm{t}\end{array}$ & $\begin{array}{c}\text { ‘tempo térmico' acumulado } \\
\text { (graus-dia) }\end{array}$ \\
\hline 1 & 1 & 15 & 5 & 10 & 10 \\
\hline 2 & 1 & 10 & & 5 & 15 \\
\hline 3 & 1 & 11 & & 6 & 21 \\
\hline 4 & 1 & 8 & & 3 & 24 \\
\hline 5 & 1 & 9 & & 4 & 28 \\
\hline 6 & 1 & 10 & & 5 & 33 \\
\hline 7 & 1 & 5 & & 0 & 33 \\
\hline 8 & 1 & 4 & & -1 & 33 \\
\hline
\end{tabular}




\section{GRAUS-DIA E GERMINAÇÃO}

\section{TEMPERATURAS SUB-ÓTIMAS}

Mesmo em condições ambientais aparentemente homogêneas, a germinação de uma população de sementes raramente é um processo simultâneo. Ao contrário, na grande maioria dos casos, a germinação é mais ou menos espalhada ao longo de um determinado intervalo de tempo (Washitani 1985). O progresso da germinação é ilustrado pelos gráficos de porcentagens de sementes germinadas contra os respectivos tempos de contagem, o que em muitos casos produz curvas como aquelas mostradas nas Figuras 2A (germinação acumulada) e 2B (germinação não acumulada). Em outras palavras, pode-se dizer que as sementes de

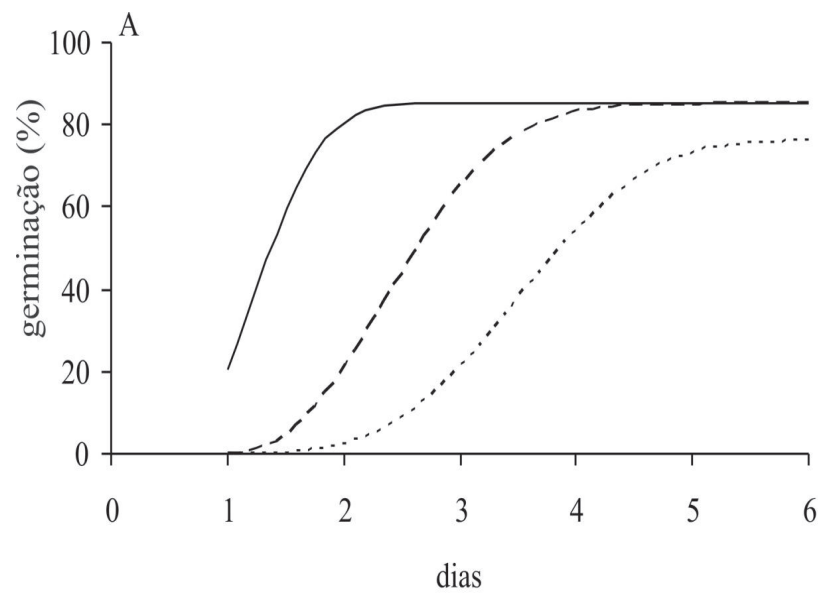

uma população germinam em tempos diferentes, ou que a distribuição dos tempos de germinação $\left(\mathrm{t}_{\mathrm{g}}\right)$ varia entre as sementes da população. Considerandose o inverso de $\mathrm{t}_{\mathrm{g}}$ como a velocidade de germinação $\left(\mathrm{V}=1 / \mathrm{t}_{\mathrm{g}}\right)$, observa-se que frequentemente $\mathrm{V}$ aumenta linearmente com a temperatura no intervalo de $T_{b}$ até $T_{o}$, decrescendo a partir daí. Quando $V=f(T)$ é linear, o ponto onde a reta intercepta o eixo $\mathrm{X}$ (Figura 3) representa $T_{b}$, onde $V=0$, de modo que a dependência térmica da velocidade de germinação pode ser representada pela equação (Garcia-Huidobro et al. 1982):

$$
\mathrm{V}=1 / \mathrm{t}=\mathrm{b} \cdot\left(\mathrm{T}-\mathrm{T}_{\mathrm{b}}\right) \quad(\text { equação } 1)
$$

onde: b é o coeficiente de inclinação da reta.

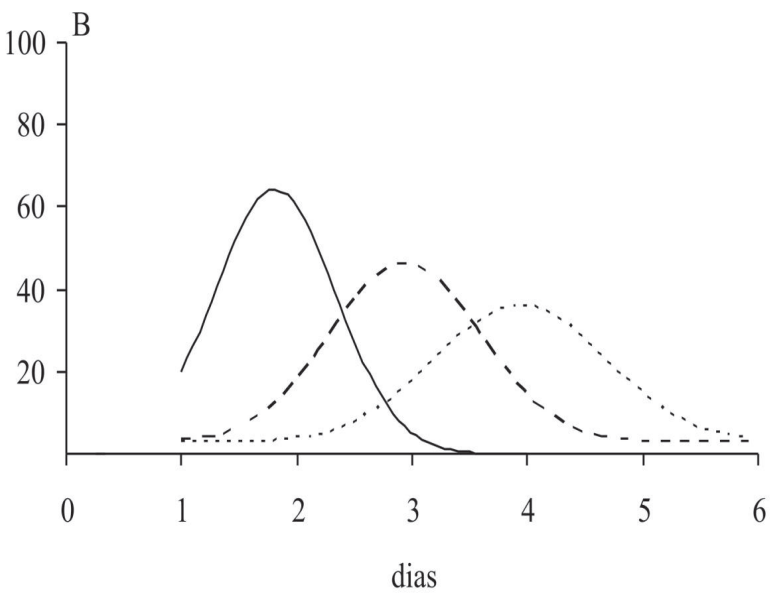

Figura 2. Exemplos hipotéticos de curvas de germinação acumulada (A) e não acumulada (B) em três diferentes temperaturas. Figure 2. Hypothetical examples of accumulated (A) and not accumulated (B) germination time courses at three temperatures.

Nesse caso, a recíproca de b é o 'tempo térmico' ( $\theta$ ) para a germinação, ou seja, $1 / b=\theta \therefore b=1 / \theta$. A grandeza $\theta$ descreve a taxa de progresso da germinação de uma população de sementes em função do aumento da temperatura $(\mathrm{T})$ a partir de $\mathrm{T}_{\mathrm{b}}$, considerando-se $\mathrm{T}<\mathrm{T}_{\mathrm{o}}$, e corresponde à quantidade de 'tempo térmico' acumulado, que pode ser descrita em termos de graus-dia (vide acima). Supondo-se que dentro de um lote as sementes estejam agrupadas em inúmeras sub-populações cujas respostas à temperatura sejam definidas pelos parâmetros $\mathrm{T}_{\mathrm{b}}$ e $\theta$, se considerarmos que cada sub-população germina sempre na mesma sequência, independentemente da temperatura, então tais sub-populações podem ser identificadas por um valor de $G$, que nada mais é do que uma determinada fração ou porcentagem de germinação acumulada. Assim, por exemplo, um valor de $G=50$ identifica uma sub-população que germina imediatamente após metade da população já ter germinado. Do mesmo modo, uma fração $G$ $=10$ representa o grupo de sementes que germina logo após os primeiros $10 \%$ das sementes terem germinado, e assim sucessivamente. Considerandose que $\mathrm{G}$ varia suavemente com o tempo, desde zero até $\mathrm{G}$ máxima, pode-se admitir um amplo espectro de sub-populações, cujos respectivos valores de $T_{b}$ e/ou $\theta$ variam de maneira praticamente contínua (GarciaHuidobro et al. 1982). 


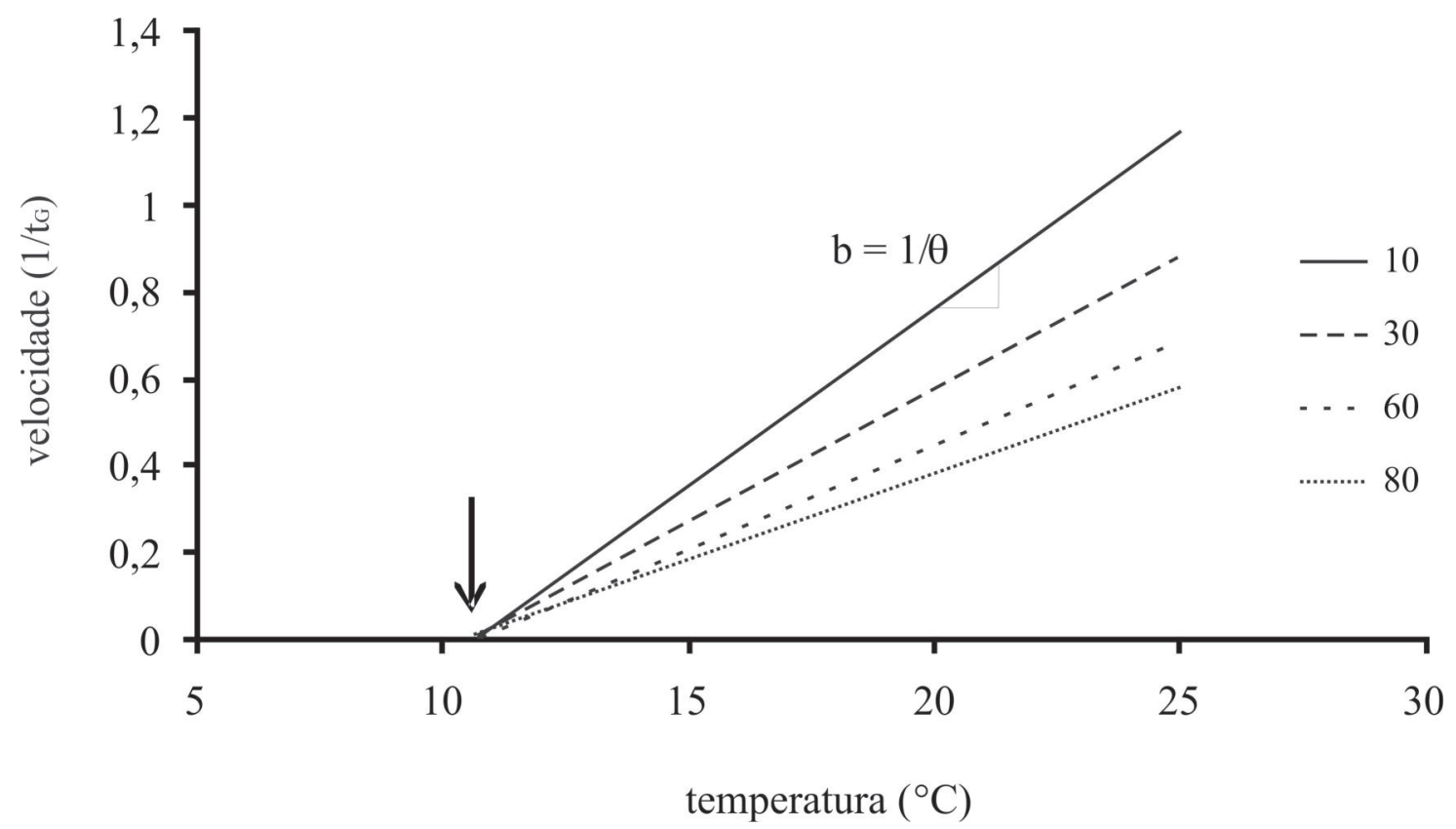

Figura 3. Relação entre velocidade de germinação e temperatura, a partir dos exemplos hipotéticos apresentados na Figura 2. As velocidades correspondem às recíprocas dos tempos de germinação estimados para as frações $10 \%(10), 30 \%(30), 60 \%(60)$ e $80 \%(80)$. Seta indica a temperatura mínima para ocorrência do processo $\left(\mathrm{T}_{\mathrm{b}}\right)$.

Figure 3. The relationship between germination rate and temperature from the data presented at Figure 2. The rates are the reciprocal of the times for germination of the fractions $10 \%(10), 30 \%(30), 60 \%(60)$ and $80 \%(80)$. The arrow shows the minimum temperature for the process occurrence $\left(\mathrm{T}_{\mathrm{b}}\right)$.

Após o trabalho pioneiro de Garcia-Huidobro et al. (1982), vários outros que investigaram o efeito da temperatura $(\mathrm{T})$ sobre a velocidade de germinação (V) obtiveram relações semelhantes à apresentada na Figura 3. Em outras palavras, se plotarmos V (inverso do tempo necessário para a germinação de uma fração $\mathrm{G}$ arbitrária, $1 / \mathrm{t}_{\mathrm{G}}$ ) contra $\mathrm{T}$, as retas correspondentes a cada fração tendem a convergir para um único ponto no eixo $\mathrm{X}$, ao passo que suas inclinações (b) diferem entre si. Embora essa relação não deva ser de caráter universal, ela indica que na grande maioria dos casos estudados $T_{b}$ é relativamente constante, ou seja, todas as sub-populações de uma amostra de sementes tem uma $\mathrm{T}_{\mathrm{b}}$ comum. Além disso, pode-se concluir que nas temperaturas da faixa infra-ótima o 'tempo térmico' $(\theta)$ varia com a sub-população, lembrando que $\theta=1 / \mathrm{b}$. A Figura 3 ilustra esse ponto, podendose observar que nas frações menores (por exemplo, $\mathrm{G}=10$ ) as relações entre $\mathrm{V}$ e $\mathrm{T}$ exibem valores de b maiores do que nas frações mais elevadas (ex.: G $=80$ ), indicando que as sub-populações identificadas pelas frações menores (ou seja, as sementes que germinam mais rapidamente) necessitam de uma quantidade acumulada de 'tempo térmico' para germinar relativamente menor do que as subpopulações formadas pelas sementes mais 'lentas' (aquelas representadas pelas frações maiores). Desse modo, para a faixa térmica infra-ótima o símbolo que representa o 'tempo térmico' é acompanhado da fração que ele identifica, representada genericamente pela letra $\mathrm{G}$, ou seja, $\theta_{\mathrm{G}}$ com $\mathrm{G}$ podendo variar entre zero e $100 \%$. Na faixa infra-ótima, portanto, a relação entre a velocidade de germinação de uma sub-população de sementes e a temperatura pode ser representada pela equação 1 reescrita da seguinte forma (Allen et al. 2007):

$$
1 / \mathrm{t}_{\mathrm{G}}=\left(1 / \theta_{\mathrm{G}}\right) \cdot\left(\mathrm{T}-\mathrm{T}_{\mathrm{b}}\right) \therefore \theta_{\mathrm{G}}=\left(\mathrm{T}-\mathrm{T}_{\mathrm{b}}\right) \cdot \mathrm{t}_{\mathrm{G}}(\text { equação } 2)
$$

De acordo com a equação 2, a quantidade de 'tempo térmico' $\left(\theta_{\mathrm{G}}\right)$ acumulado para a germinação de uma fração percentual $\mathrm{G}$ de sementes é o produto do tempo 'real' para a germinação dessa porcentagem pela diferença entre a temperatura ambiente e a temperatura base (ou mínima). Sendo $\theta_{\mathrm{G}}$ constante para uma dada fração $\mathrm{G}$, o tempo necessário para $\mathrm{G} \%$ das sementes germinarem $\left(\mathrm{t}_{\mathrm{G}}\right.$ ) é inversamente proporcional à diferença entre $\mathrm{T}$ e $\mathrm{T}_{\mathrm{b}}$. Assim, quanto 
maior a diferença entre $\mathrm{T}$ e $\mathrm{T}_{\mathrm{b}}$ menor o tempo para a germinação. Isso implica que, na faixa infra-ótima, o 'tempo térmico' (grau-dia ou grau-hora) para a germinação de uma dada sub-população é o mesmo em qualquer temperatura (Allen et al. 2007). Nesse caso, a distribuição dos tempos de germinação entre as sementes de uma população pode ser atribuída à variação na quantidade de 'tempo térmico' necessário à germinação de cada semente, sendo que em alguns casos essa distribuição de $\theta_{\mathrm{G}}$ é assumida como sendo normal, podendo assim ser caracterizada por uma média $(\mu)$ e um desvio padrão $(\sigma)$ (Figura 4). Essa variação na distribuição de $\theta_{G}$, portanto, produziria a característica curva sigmóide das curvas de germinação (Figura 2). Como reportam Garcia-Huidobro et al. (1982), a temperatura deve simplesmente fazer com que a distribuição das germinações seja mais agrupada (sincronizada) ou espalhada ao longo do tempo, sem contudo alterar de maneira significativa a ordem da distribuição dos períodos de germinação, ou seja, uma sub-população de sementes 'rápidas' sempre tenderá a germinar antes de uma sub-população de sementes 'lentas' ou menos rápidas, independentemente da temperatura.

No exemplo de modelagem da germinação usando o conceito de 'tempo térmico' (vide abaixo), assumiremos esses pressupostos, com $\mathrm{T}_{\mathrm{b}}$ constante para todas as sementes e $\theta_{\mathrm{G}}$ variando normalmente entre diferentes sub-populações. Entretanto, podem existir casos em que $T_{b}$ varia dentro de um lote de sementes (Kebreab \& Murdoch 2000), principalmente se essas apresentarem dormência. Nesses casos, adaptações são feitas ao modelo para se descrever o progresso da germinação (Kruk \& Benech-Arnold 1998, Batlla \& Benech-Arnold 2003), mas isso não será tratado no presente artigo. Para se saber se um lote de sementes apresenta uma única $T_{b}$ ou se essa varia dentro da população, deve-se plotar $1 / t_{\mathrm{G}}$ como uma função de $\mathrm{T}$ para diferentes valores de $\mathrm{G}$ (por exemplo, $10 \%, 50 \%$ e $80 \%$ ) e verificar se as retas de regressão convergem para um ponto comum no eixo da temperatura, conforme a Figura 3. Para se estimar $t_{G}$, por sua vez, o procedimento é plotar-se as porcentagens de germinação acumulada contra os respectivos tempos e traçar uma curva que descreva da melhor maneira possível a distribuição dos pontos, ou simplesmente unir os pontos com segmentos de reta e estimar os tempos necessários para um dado valor de $G$ por interpolação. Simão et al. (2010) usaram a função de Weibull (Dumur et al. 1990) para descrever as curvas de germinação em diferentes temperaturas e estimar $t_{G}$ :

$$
\begin{aligned}
\mathrm{G} & =\mathrm{M}\left\{1-\exp \left[-\mathrm{k}\left(\mathrm{t}_{\mathrm{G}}-\mathrm{z}\right)^{\mathrm{c}}\right]\right\} \quad \therefore \\
\mathrm{t}_{\mathrm{G}} & =\left\{\left[\ln (\mathrm{M} /(\mathrm{M}-\mathrm{G}))^{1 / \mathrm{c}}\right] / \mathrm{k}\right\}+\mathrm{z}
\end{aligned}
$$

onde: $\mathrm{G}$ é a porcentagem de germinação acumulada (ordenada) no tempo $\mathrm{t}_{\mathrm{G}}$ (abscissa). $\mathrm{M}, \mathrm{k}, \mathrm{z}$ e c são constantes calculadas por qualquer programa de ajuste de curvas que contenha a função de Weibull.

Além do método gráfico mencionado acima, o valor de $\mathrm{T}_{\mathrm{b}}$ pode ser estimado por outros métodos (Steinmaus et al. 2000), entre eles o de análise de regressão probítica (Bradford 1995), o qual será tratado neste artigo. De qualquer modo, é recomendávelusar-se primeiramenteométodográfico para verificar se podemos assumir uma única $\mathrm{T}_{\mathrm{b}}$ para as diferentes sub-populações. Considerando-se que a distribuição de $\theta_{\mathrm{G}}$ seja normal para as diferentes frações $G$ na população, pode-se proceder à análise. Inicialmente, é preciso transformar as porcentagens acumuladas de germinação correspondentes a cada dia de contagem em probit (recurso INV. NORM da planilha eletrônica Excel), procedimento esse levado a cabo para as temperaturas subótimas. Os valores transformados de todas as temperaturas são então plotados contra $\log \theta_{\mathrm{G}}=\log \left[\left(\mathrm{T}-\mathrm{T}_{\mathrm{b}}\right) \mathrm{t}_{\mathrm{G}}\right]$ num único gráfico. A Tabela 2 ilustra como deve ser montada a planilha eletrônica para a elaboração do gráfico único, com $\log \theta_{\mathrm{G}}$ no eixo $\mathrm{X}$ e os respectivos probits $(\mathrm{G})$ no eixo Y. A equação que descreve essa relação é a seguinte (Ellis et al. 1986):

$$
\begin{aligned}
& \operatorname{probit}(\mathrm{G})=a+\left[\log \left(\mathrm{T}-\mathrm{T}_{\mathrm{b}}\right) \mathrm{t}_{\mathrm{G}}\right] / \sigma_{\theta} \quad \therefore \\
& \operatorname{probit}(\mathrm{G})=a+\left(\log \theta_{\mathrm{G}}\right) / \sigma_{\theta}(\text { equação 3) }
\end{aligned}
$$

onde: $\sigma_{\theta}$ - representado pelo inverso do coeficiente de inclinação da reta - é o desvio padrão da distribuição dos valores de $\theta_{\mathrm{G}}$ requeridos pelas várias sub-populações; T é a temperatura experimental; e $a$ é o intercepto (Figura 4A). 

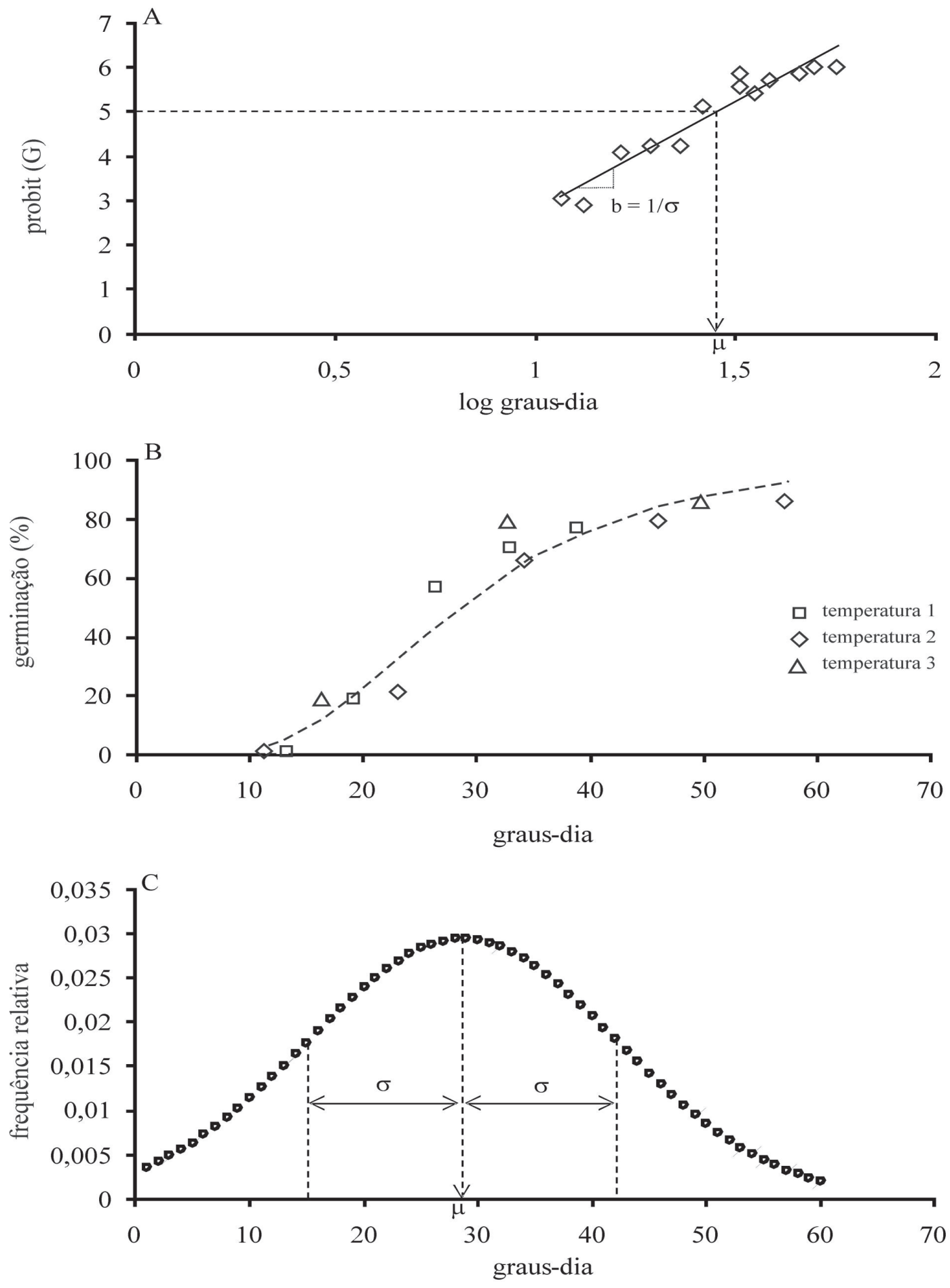

Figura 4. Parâmetros da distribuição do 'tempo térmico' $\left(\theta_{\mathrm{G}}\right)$ necessário para a germinação de um lote de sementes. A: Análise probítica das curvas de germinação hipotéticas apresentadas na Figura 2, onde as porcentagens de germinação nas três temperaturas foram transformadas em probit e plotadas contra $\log \left(\mathrm{T}-\mathrm{T}_{\mathrm{b}}\right) \mathrm{t}_{\mathrm{G}}$ (ver texto para detalhes). Diferentes temperaturas mínimas para ocorrência do processo $\left(\mathrm{T}_{\mathrm{b}}\right)$ foram experimentados até se obter o

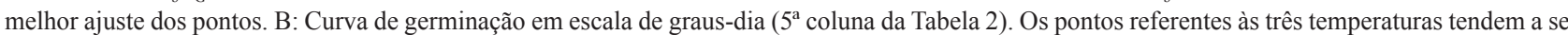
posicionar na mesma linha, que representa a curva teórica obtida a partir dos parâmetros média $(\mu)$ e desvio padrão $(\sigma)$ do modelo. C: A mesma curva apresentada em B, mas plotada em termos de distribuição normal de frequências de $\theta_{\mathrm{G}}$, a partir dos parâmetros $\mu$ e $\sigma$.

Figure 4. Parameters of the distribution of thermal time $\left(\theta_{\mathrm{d}}\right)$ required for germination of a seed batch. A: Probitic analisys of the hypothetical germination courses presented at Figure 2, where the germination percentages at the three temperatures were probit transformed and plotted against $\log \left(\mathrm{T}_{\mathrm{b}} \mathrm{\textrm {b }} \mathrm{t}_{\mathrm{G}}\right.$ (see text for details). Different values of the base temperature for germination $\left(\mathrm{T}_{\mathrm{b}}\right)$ were tried until the best fit. B: The germination time courses in thermal time scale ( $5^{\text {th }}$ column of the Table 2). The points concerning the three temperatures tend to fall on the same line which represents the theoretical curve obtained from the model parameters mean $(\mu)$ and standard deviation $(\sigma)$. C: The same curve presented in B is plotted in terms of the normal distribution of the relative frequencies of $\theta_{\mathrm{G}}$ from the parameters $\mu$ and $\sigma$. 
Uma vez gerada a reta de regressão de $\operatorname{probit}(\mathrm{G})$ (última coluna da Tabela 2$)$ em $\log \theta_{\mathrm{G}}\left(6^{\mathrm{a}}\right.$ coluna), diferentes valores de $T_{b}\left(4^{a}\right.$ coluna) são experimentados sucessivamente até se obter o melhor ajuste da curva (maior $\mathrm{R}^{2}$ ) que descreve o modelo (equação 3) (Bradford 1995). Obtém-se desse modo a média $(\mu)$, que é o valor no eixo $X$ correspondente ao probit 5 (= $50 \%$ da germinação) no eixo Y, e o desvio padrão $(\sigma)$, que é o inverso da inclinação $b$ (Figura 4A). Esses ( $\mu$ e $\sigma$ ) são, portanto, os parâmetros que descrevem a distribuição de $\theta_{\mathrm{G}}$ dentro da população, assumindose que a mesma seja normal (Figura 4C). A Figura 4B ilustra como as curvas apresentadas na Figura 2 caem aproximadamente na mesma linha quando plotadas em escala de 'tempo térmico' (graus-dia), mostrando que o principal efeito da temperatura na faixa sub-ótima é retardar e tornar a germinação mais espalhada ao longo do tempo, sem, contudo, interferir na ordem de germinação das sementes. Assim, tendose determinado $\mathrm{T}_{\mathrm{b}}$ para um lote de sementes ou para uma população, e conhecendo-se a quantidade total de 'tempo térmico' necessário para a germinação, o tempo real para a que a semente germine numa temperatura qualquer pode ser estimado a partir da equação 3, rearranjada da seguinte forma:

$$
\mathrm{t}_{\mathrm{G}}=\left[10^{\text {(probit(G)-a). } \sigma}\right] /\left(\mathrm{T}-\mathrm{T}_{\mathrm{b}}\right)
$$

Tabela 2. Exemplo de arranjo de uma planilha para estimativa da temperatura base $\left(\mathrm{T}_{\mathrm{b}}\right)$ pelo método de regressão probítica. Os dados referem-se à um ensaio hipotético realizado em três temperaturas infra-ótimas (I, II e III) e cujas porcentagens acumuladas de germinação (G) foram registradas durante quatro dias $\left(\mathrm{G} 1, \mathrm{G} 2, \mathrm{G} 3\right.$ e G4). Onde $\mathrm{q}_{\mathrm{G}}=\left(\mathrm{T}-\mathrm{T}_{\mathrm{b}}\right) \cdot \mathrm{t}_{\mathrm{G}}$ ('tempo térmico') e $\mathrm{T}$ indica a temperatura experimental.

Table 2. Example of electronic worksheet for estimating the base temperature ( $\mathrm{T}_{\mathrm{b}}$ ) by the probit regression method. The data concern to a hypothetical assay at three infra-optimal temperatures (I, II and III) whose cumulative germination percentages ( $G$ ) were recorded for four days (G1, G2, G3 and G4). Where $\mathrm{q}_{\mathrm{G}}=\left(\mathrm{T}-\mathrm{T}_{\mathrm{b}}\right) \cdot \mathrm{t}_{\mathrm{G}}$ (thermal time) and Tindicates the experimental temperature.

\begin{tabular}{ccccccc}
\hline tempo $\left(\mathrm{t}_{\mathrm{G}}\right)$ & $\mathrm{G}$ & $\mathrm{T}$ & $\mathrm{T}_{\mathrm{b}}$ & $\theta_{\mathrm{G}}$ & $\log \theta_{\mathrm{G}}$ & probit $(\mathrm{G})$ \\
\hline 1 & $\mathrm{G} 1$ & $\mathrm{~T}_{\mathrm{I}}$ & $\mathrm{T}_{\mathrm{b}}$ & $\theta_{\mathrm{G} 1}$ & $\log \theta_{\mathrm{G} 1}$ & probitG1 \\
2 & $\mathrm{G} 2$ & $\mathrm{~T}_{\mathrm{I}}$ & & $\theta_{\mathrm{G} 2}$ & $\log \theta_{\mathrm{G} 2}$ & probitG2 \\
3 & $\mathrm{G} 3$ & $\mathrm{~T}_{\mathrm{I}}$ & & $\theta_{\mathrm{G} 3}$ & $\log \theta_{\mathrm{G} 3}$ & probitG3 \\
4 & $\mathrm{G} 4$ & $\mathrm{~T}_{\mathrm{I}}$ & & $\theta_{\mathrm{G} 4}$ & $\log \theta_{\mathrm{G} 4}$ & probitG4 \\
1 & $\mathrm{G} 1$ & $\mathrm{~T}_{\mathrm{II}}$ & $\theta_{\mathrm{G} 1}$ & $\log \theta_{\mathrm{G} 1}$ & probitG1 \\
2 & $\mathrm{G} 2$ & $\mathrm{~T}_{\mathrm{II}}$ & $\theta_{\mathrm{G} 2}$ & $\log \theta_{\mathrm{G} 2}$ & probitG2 \\
3 & $\mathrm{G} 3$ & $\mathrm{~T}_{\mathrm{II}}$ & $\theta_{\mathrm{G} 3}$ & $\log \theta_{\mathrm{G} 3}$ & probitG3 \\
4 & $\mathrm{G} 4$ & $\mathrm{~T}_{\mathrm{II}}$ & $\theta_{\mathrm{G} 4}$ & $\log \theta_{\mathrm{G} 4}$ & probitG4 \\
1 & $\mathrm{G} 1$ & $\mathrm{~T}_{\mathrm{III}}$ & $\theta_{\mathrm{G} 1}$ & $\log \theta_{\mathrm{G} 1}$ & probitG1 \\
2 & $\mathrm{G} 2$ & $\mathrm{~T}_{\mathrm{III}}$ & $\theta_{\mathrm{G} 2}$ & $\log \theta_{\mathrm{G} 2}$ & probitG2 \\
3 & $\mathrm{G} 3$ & $\mathrm{~T}_{\text {III }}$ & $\theta_{\mathrm{G} 3}$ & $\log \theta_{\mathrm{G} 3}$ & probitG3 \\
4 & $\mathrm{G} 4$ & $\mathrm{~T}_{\mathrm{III}}$ & $\theta_{\mathrm{G} 4}$ & $\log \theta_{\mathrm{G} 4}$ & probitG4 \\
\hline
\end{tabular}

\section{TEMPERATURAS SUPRA-ÓTIMAS}

De acordo com a equação 2, a velocidade de germinação continuaria a aumentar indefinidamente com o aumento da temperatura, o que evidentemente não ocorre em processos com uma temperatura (ou intervalo térmico) ótima seguida de uma faixa térmica na qual a velocidade decresce com o aumento de $\mathrm{T}$ até um valor limítrofe representado por $\mathrm{T}_{c}$, a partir do qual a germinação deixa de ocorrer (Allen et al. 2007). Têm-se observado em alguns casos que, em temperaturas supra-ótimas, $\mathrm{T}_{\mathrm{c}}$ varia entre as sementes, enquanto que a quantidade de 'tempo térmico' $(\theta)$ acumulado para que a germinação ocorra assume valor relativamente constante (Bradford 2002) (Figura 5). Entretanto, assim como no caso 
de $T_{b}$, parece que isso nem sempre ocorre, havendo relatos de que diferentes sub-populações de uma amostra podem apresentar uma $\mathrm{T}_{\mathrm{c}}$ comum (GarciaHuidobro et al. 1982). No primeiro caso, ou seja, quando $T_{c}$ varia dentro da população (Figura 5), as curvas de germinação na faixa supra-ótima podem ser analisadas considerando-se $\theta$ constante e assumindose que $T_{c}$ varia entre as diferentes sub-populações, de acordo com o seguinte modelo (Ellis \& Butcher 1988):

$$
\begin{gathered}
\theta=\left(\mathrm{T}_{\mathrm{c}(\mathrm{G})}-\mathrm{T}\right) \mathrm{t}_{\mathrm{G}} \therefore 1 / \mathrm{t}_{\mathrm{G}}=\left(\mathrm{T}_{\mathrm{c}(\mathrm{G})}-\mathrm{T}\right) / \theta \\
\text { (equação } 4)
\end{gathered}
$$

onde: $\theta$ é a constante de 'tempo térmico' para a faixa supra-ótima; $\mathrm{T}_{\mathrm{c}(\mathrm{G})}$ é a temperatura máxima para a germinação da sub-população identificada pela fração percentual $G$; $t_{G}$ é o tempo para a germinação da fração $\mathrm{G}$; e $\mathrm{T}$ é a temperatura experimental.

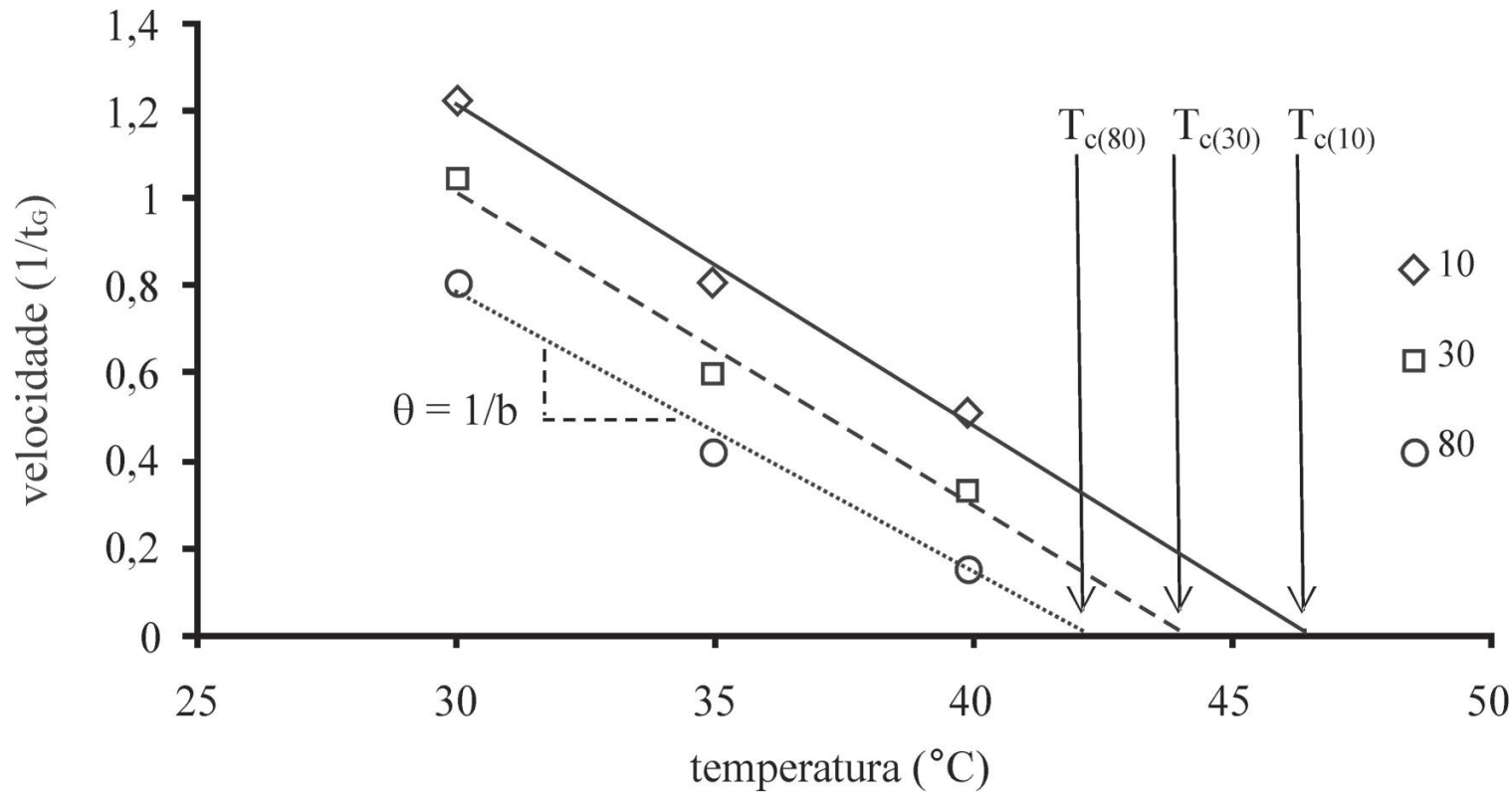

Figura 5. Exemplo hipotético da relação entre temperaturas supra-ótimas (de $30^{\circ} \mathrm{C}$ a $\left.40^{\circ} \mathrm{C}\right)$ e o inverso do tempo para germinação de $10 \%(\diamond), 30 \%$ $(\square)$ e $80 \%(\circ)$ das sementes. $T_{c(G)}$ indica a temperatura máxima para germinação da fração percentual arbitrária $\mathrm{G}$ (no caso $10 \%, 30 \%$ ou $80 \%$ ); $\theta$ é a constante de 'tempo térmico', correspondendo ao inverso das inclinações das retas.

Figure 5. Hypothetical example of the relationship amongst supra-optimal temperatures (from $30^{\circ} \mathrm{C}$ to $40^{\circ} \mathrm{C}$ ) and the inverse of the time for germination of $10 \%(\diamond), 30 \%(\square)$ and $80 \%(0)$ of the seeds. $\mathrm{T}_{\mathrm{c}(\mathrm{G})}$ indicates the ceiling temperature concerning an arbitrary per cent fraction $\mathrm{G}($ ex. $10 \%, 30 \%$ or $80 \%) ; \theta$ is the thermal time constant, corresponding to the inverse of the slopes of the lines.

De acordo com a equação 4 , sendo $\theta$ constante para todas as sementes, a variação nos tempos de germinação de diferentes sub-populações decorreria da diferença entre $T_{c}$ e a temperatura experimental $T$. Em outras palavras, $\mathrm{T}_{\mathrm{c}}$ seria um parâmetro intrínseco de cada semente, sendo portanto representado pelo símbolo $\mathrm{T}_{\mathrm{c}(\mathrm{G})}$, e a velocidade de germinação de cada semente numa temperatura supra-ótima decorreria das variações individuais nos valores desse parâmetro. Assim, para uma dada temperatura $\mathrm{T}$ supra-ótima, a diferença nos tempos de germinação entre duas sementes da amostra seria devido às suas respectivas $\mathrm{T}_{\mathrm{c}(\mathrm{G})}$. Quanto mais afastada estiver $\mathrm{T}_{\mathrm{c}(\mathrm{G})}$ de $\mathrm{T}$, maior será a velocidade da germinação (Allen et al. 2007). Assim como no caso de $\theta_{\mathrm{G}}$ na faixa infra-ótima, a variação de $T_{c(G)}$ é em muitos casos normalmente distribuída dentro da população de sementes, permitindo assim que a germinação seja modelada a partir dos parâmetros $\mu$ (média) e $\sigma$ (desvio padrão) (Bradford 2002).

O procedimento para a estimativa de $\mu$ e $\sigma$ é similar ao utilizado para as temperaturas da faixa infra-ótima. Os dados das curvas de germinação (porcentagens acumuladas contra os respectivos tempos) das temperaturas supra-ótimas são organizados conjuntamente numa única planilha, segundo o modelo apresentado na Tabela 3. Tendo-se gerado a reta de regressão dos valores de probit(G) (última coluna da Tabela 3$)$ em $\log \mathrm{T}_{\mathrm{c}(\mathrm{G})}\left(6^{\mathrm{a}}\right.$ coluna), diferentes valores de $\theta$ ( $4^{\mathrm{a}}$ coluna) são experimentados sucessivamente até se obter o maior valor de $\mathrm{R}^{2}$, de acordo com seguinte modelo (Ellis et al. 1986): 


$$
\begin{aligned}
& \operatorname{probit}(\mathrm{G})=\mathrm{a}+\log \left(\mathrm{T}+\theta / \mathrm{t}_{\mathrm{G}}\right) / \sigma_{\mathrm{Tc}} \\
& \operatorname{probit}(\mathrm{G})=\mathrm{a}+\log \mathrm{T}_{\mathrm{c}(\mathrm{G})} / \sigma_{\mathrm{Tc}}(\text { equação } 5)
\end{aligned}
$$

onde: $\operatorname{probit}(\mathrm{G})$ é a transformação probítica da porcentagem G; a é o intercepto do eixo X; $\sigma_{\text {Tc }}$ é o desvio padrão da distribuição de $T_{c}$ na população.

Tabela 3. Exemplo de arranjo de uma planilha para estimativa do 'tempo térmico' $\left(\theta=\left(\mathrm{T}_{\mathrm{c}(\mathrm{G})}-\mathrm{T}\right) \cdot \mathrm{t}_{\mathrm{G}}\right)$ e da temperatura máxima para germinação de uma fração da população de sementes $\left(\mathrm{T}_{\mathrm{c}(\mathrm{G})}\right)$ pelo método de regressão probítica. Os dados referem-se às porcentagens acumuladas de germinação registradas durante quatro dias (G1, G2, G3 e G4), em três temperaturas supra-ótimas (IV, V e VI). Onde: G é a porcentagem acumulada de germinação; T é a temperatura experimental; e $\mathrm{T}_{\mathrm{c}(\mathrm{G})}$ é temperatura máxima para a germinação de uma fração $\mathrm{G}$.

Table 3. Example of electronic worksheet for estimating the thermal time $\left(\theta=\left(\mathrm{T}_{\mathrm{c}(\mathrm{G})}-\mathrm{T}\right) . \mathrm{t}_{\mathrm{G}}\right)$ and the ceiling temperature for a given fraction of the seed population $\left(\mathrm{T}_{\mathrm{c}(\mathrm{G})}\right)$ by the probit regression method. The data concern to the cumulative daily germination percentages recorded for four days (G1, G2, G3 and $\mathrm{G} 4)$, at three supra-optimal temperatures (IV, $\mathrm{V}$ and $\mathrm{VI})$. Where: $\mathrm{G}$ is the cumulative germination percentage; $\mathrm{T}$ is the experimental temperature;

\begin{tabular}{|c|c|c|c|c|c|c|}
\hline tempo $\left(t_{G}\right)$ & G & $\mathrm{T}$ & $\mathrm{q}$ & $\mathrm{T}_{\mathrm{c}(\mathrm{G})}$ & $\log \mathrm{T}_{\mathrm{c}(\mathrm{G})}$ & probit $(\mathrm{G})$ \\
\hline 1 & G1 & $\mathrm{T}_{\mathrm{IV}}$ & $\mathrm{q}$ & $\mathrm{T}_{\mathrm{c}(\mathrm{G} 1)}$ & $\log \mathrm{T}_{\mathrm{c}(\mathrm{G} 1)}$ & probitG1 \\
\hline 2 & G2 & $\mathrm{T}_{\mathrm{IV}}$ & & $\mathrm{T}_{\mathrm{c}(\mathrm{G} 2)}$ & $\log \mathrm{T}_{\mathrm{c}(\mathrm{G} 2)}$ & probitG2 \\
\hline 3 & G3 & $\mathrm{T}_{\mathrm{IV}}$ & & $\mathrm{T}_{\mathrm{c}(\mathrm{G} 3)}$ & $\log \mathrm{T}_{\mathrm{c}(\mathrm{G} 3)}$ & probitG3 \\
\hline 4 & G4 & $\mathrm{T}_{\mathrm{IV}}$ & & $\mathrm{T}_{\mathrm{c}(\mathrm{G} 4)}$ & $\log \mathrm{T}_{\mathrm{c}(\mathrm{G} 4)}$ & probitG4 \\
\hline 1 & G1 & $\mathrm{T}_{\mathrm{v}}$ & & $\mathrm{T}_{\mathrm{c}(\mathrm{G} 1)}$ & $\log \mathrm{T}_{\mathrm{c}(\mathrm{Gl})}$ & probitG1 \\
\hline 2 & $\mathrm{G} 2$ & $\mathrm{~T}_{\mathrm{v}}$ & & $\mathrm{T}_{\mathrm{c}(\mathrm{G} 2)}$ & $\log \mathrm{T}_{\mathrm{c}(\mathrm{G} 2)}$ & probitG2 \\
\hline 3 & G3 & $\mathrm{T}_{\mathrm{v}}$ & & $\mathrm{T}_{\mathrm{c}(\mathrm{G} 3)}$ & $\log \mathrm{T}_{\mathrm{c}(\mathrm{G} 3)}$ & probitG3 \\
\hline 4 & G4 & $\mathrm{T}_{\mathrm{v}}$ & & $\mathrm{T}_{\mathrm{c}(\mathrm{G} 4)}$ & $\log \mathrm{T}_{\mathrm{c}(\mathrm{G} 4)}$ & probitG4 \\
\hline 1 & G1 & $\mathrm{T}_{\mathrm{VI}}$ & & $\mathrm{T}_{\mathrm{c}(\mathrm{G} 1)}$ & $\log \mathrm{T}_{\mathrm{c}(\mathrm{G} 1)}$ & probitG1 \\
\hline 2 & $\mathrm{G} 2$ & $\mathrm{~T}_{\mathrm{VI}}$ & & $\mathrm{T}_{\mathrm{c}(\mathrm{G} 2)}$ & $\log \mathrm{T}_{\mathrm{c}(\mathrm{G} 2)}$ & probitG2 \\
\hline 3 & G3 & $\mathrm{T}_{\mathrm{VI}}$ & & $\mathrm{T}_{\mathrm{c}(\mathrm{G} 3)}$ & $\log \mathrm{T}_{\mathrm{c}(\mathrm{G} 3)}$ & probitG3 \\
\hline 4 & G4 & $\mathrm{T}_{\mathrm{VI}}$ & & $\mathrm{T}_{\mathrm{c}(\mathrm{G} 4)}$ & $\log \mathrm{T}_{\mathrm{c(G4)}}$ & probitG4 \\
\hline
\end{tabular}
and $\mathrm{T}_{\mathrm{c}(\mathrm{G})}$ is the ceiling temperature concerning a fraction $G$.

Assim como no caso do gráfico para estimativa de $\mathrm{T}_{\mathrm{b}}$, a média $(\mu)$ corresponde ao probit $=5$ e o desvio padrão $(\sigma)$ é o inverso da inclinação (b) da reta. Com esses parâmetros ( $\mu$ e $\sigma$ ) descritores da distribuição de $T_{c(G)}$ na população pode-se estimar o tempo real $\left(\mathrm{t}_{\mathrm{G}}\right)$ para a germinação de acordo com a equação 5 rearranjada:

$$
\mathrm{t}_{\mathrm{G}}=\theta /\left[10^{(\text {probit(G)-a). } \sigma-\mathrm{T}}\right]
$$

\section{CONSIDERAÇÕES FINAIS}

Os procedimentos descritos nesta pequena revisão buscam explicar o efeito da temperatura sobre a distribuição dos tempos de germinação, assumindose que as sementes da amostra apresentam uma temperatura base $\left(\mathrm{T}_{\mathrm{b}}\right)$ comum e que a quantidade de 'tempo térmico' acumulada não varia para as temperaturas do intervalo supra-ótimo. Desse modo, os parâmetros que determinam a distribuição dos tempos de germinação de sementes individuais em temperaturas infra e supra-ótimas seriam, respectivamente, $\theta_{\mathrm{G}}$ e $\mathrm{T}_{\mathrm{c}(\mathrm{G})}$ (Ellis et al. 1986). No modelo discutido aqui, assume-se que a distribuição desses parâmetros seja normal, mas Ellis et al. (1986) postularam que esse procedimento pode ser adaptado para outros tipos de distribuição. Mais do que descrever quantitativamente a resposta de diferentes populações ou espécies à temperatura, o modelo é passível de teste e fornece um arcabouço conceitual para investigações sobre o significado fisiológico dos parâmetros ou sobre fatores que possam eventualmente alterá-los. Trata-se também de um método relativamente simples para a triagem de germoplasma, bastando que o material seja testado em duas temperaturas em cada faixa térmica (infra e supra- 
ótima) para que os parâmetros possam ser estimados. Por outro lado, Ellis et al. (1986) destacaram que as curvas de germinação corrigidas por $\theta_{\mathrm{G}} \mathrm{e} \mathrm{Tc}_{(\mathrm{G})}$ valem apenas para o lote de sementes a partir do qual os parâmetros foram obtidos, uma vez que a velocidade de germinação depende de outros fatores como o genótipo e as condições ambientais durante a maturação da semente. Os mesmos fundamentos usados no modelo de graus-dia podem ser estendidos ao fator água (hydrotime e hydrothermal time), mas isso será objeto de outro artigo.

\section{REFERÊNCIAS}

ALLEN, P.S.; BENECH-ARNOLD, R.L.; BATLLA, D. \& BRADFORD, K. 2007. Modeling of seed dormancy. Pp. 72112. In: K. Bradford \& H. Nonogaki (eds.). Seed Development, Dormancy and Germination. Annual Plant Reviews, Vol. 27. Blackwell Publishing, Oxford. 367p.

ALVARADO, V. \& BRADFORD, K.J. 2002. A hydrothermal time model explains the cardinal temperatures for seed germination. Plant, Cell and Environment, 25: 1061-1069.

BATLLA, D. \& BENECH-ARNOLD, R.L. 2003. A quantitative analysis of dormancy loss dynamics in Polygonum aviculare $L$. seeds: development of a thermal time model based on changes in seed population thermal parameters. Seed Science and Research, 13: 56-68.

BRADFORD, K. 1995. Water relations in seed germination. Pp. 351-396. In: J. Kigel \& G. Galili (eds.). Seed Development and Germination. Marcel Dekker Inc, New York. 853p.

BRADFORD, K. 2002. Applications of hydrothermal time to quantifying and modeling seed germination and dormancy. Weed Science, 50: 248-260.

CARDOSO, V.J.M. 2008. Germinação. Pp. 386-408. In: G.B. Kerbauy (org.). Fisiologia Vegetal. Guanabara Koogan, Rio de Janeiro. 431p.

DUMUR, D.; PILBEAM, C.J. \& CRAIGON, J. 1990. Use of the Weibull function to calculate cardinal temperatures in faba bean. Journal of Experimental Botany, 41: 1423-1430.

ELLIS, R.H. \& BUTCHER, P.D. 1988. The effects of priming and natural differences in quality amongst onion seed lots on the response of the rate of germination to temperature and the identification of the characteristics under genotypic control. Journal of Experimental Botany, 39: 935-950.
ELLIS, R.H.; COVELL, S.; ROBERTS, E.H. \& SUMMERFIELD, R.J. 1986. The influence of temperature on seed germination rate in grain legumes. II. Intraspecific variation in chickpea (Cicer arietinum L.) at constant temperatures. Journal of Experimental Botany, 17: 1503-1515.

GARCIA-HUIDOBRO, J.; MONTEITH, J.L. \& SQUIRE, G.R. 1982. Time, temperature and germination of pearl millet (Pennisetum typhoydes). Journal of Experimental Botany, 33: 288-296.

KEBREAB, E. \& MURDOCH, A.J. 2000. The effect of water stress on the temperature range for germination of Orobanche aegyptiaca seeds. Seed Science Research, 10: 127-133.

KRUK, B.C. \& BENECH-ARNOL, R.L. 1998. Functional and quantitative analysis of seed thermal responses in prostrate knotweed (Polygonum aviculare) and common purslane (Portulaca oleraceae). Weed Science, 46: 83-90.

LABOURIAU, L.G. 1970. On the physiology of seed germination in Vicia graminea I. Anais da Academia Brasileira de Ciências, 42: 235-262.

LABOURIAU, L.G. 1972. On the physiology of seed germination in Vicia graminea Sm. II. An analysis of the temperature dependence of the seed germination rate. Anais da Academia Brasileira de Ciências, 44: 477-534.

LABOURIAU, L.G. 1983. A Germinação das Sementes. Secretaria Geral da OEA, Washington. 174p.

MANIERO, M.A. 1980. Aplicação do método de graus-dia em cana-de-açúcar (Saccharum spp). Dissertação de Mestrado. Escola Superior de Agricultura Luiz de Queiroz - Universidade de São Paulo, Piracicaba, SP, Brasil. 75p.

MEYER, S.E.; DEBAENE-GILL, S.B. \& ALLEN, P.S. 2000. Using hydrothermal time concepts to model seed germination response to temperature, dormancy loss and priming effects in Elymus elymoides. Seed Science Research, 10: 213-223.

SIMÃO, E.; TAKAKI, M. \& CARDOSO, V.J.M. 2010. Germination response of Hylocereus setaceus (Salm-Dyck ex DC.) Ralf Bauer (Cactaceae) seeds to temperature and reduced water potentials. Brazilian Journal of Biology, 70: 135-144.

STEINMAUS, S.J.; PRATHER, T.S. \& HOLT, J.S. 2000. Estimation of base temperatures for nine weed species. Journal of Experimental Botany, 51: 275-286.

UC IPM Online. Statewide Integrated Pest Management Program, Agriculture and Natural Resources, University of California. 
2003. Degree-Days. <http://www.ipm.ucdavis.edu/WEATHER/ ddconcepts.html >. (Acesso em 20/01/2010).

WASHITANI, I. 1985. Germinatin-rate dependency on temperature of Geranium carolinianum seeds. Journal of Experimental Botany, 36: 330-337.

Submetido em 16/09/2010 Aceito em 20/12/2010 